\title{
AN UNIFIED DESIGN METHOD OF MARITIME WORKS AGAINST WAVES
}

\author{
Clavero, M. ${ }^{1}$, Vílchez, M. ${ }^{1}$, Pérez, D. ${ }^{2}$, Benedicto, M. I. ${ }^{2}$, Losada, M.A. ${ }^{1}$
}

\begin{abstract}
This work studies the interaction of a composite breakwater and irregular waves as a function of the breakwater geometry and wave characteristics. For this, tests in a flume wave were carried out. The model consisted of a composite breakwater with rectangular cross section and with the width, foundation depth and stone diameter varying in order to know their influence in the breakwater behavior. Results show that reflection coefficient increase with $B / L$ (where $B$ is the width of the dike and $L$ is the wave length) until a maximum value (saturation point) from which it is constant or decreases slightly. Before this point, reflection coefficient depends on breakwater geometry and wave characteristics and when the dike reaches the saturation regime, it only depends on stone diameter or porosity. The value of the sea water elevation at the wall and the horizontal pressure depends on the reflection coefficient. Finally, the relationship between horizontal and vertical forces is linear depending on the reflection regime. This analysis shows that energy distribution is the main variable to know the structural behavior of a dike. Based on these results, a methodology to obtain the loads over the composite breakwater is proposed.
\end{abstract}

Keywords: composite breakwater; reflection coefficient; porous media; design method

\section{INTRODUCTION}

For many decades the harbors have been protected from the action of waves by means of mound, vertical or composite breakwaters. The typology election depends on the availability of materials and constructive elements, the local experience, the cost and the possibility to justify its stability.

In case of mound breakwaters, the calculation begins applying an empirical formula (Losada and Gimenez - Curto 1979; Van der Meer 1992) that provides the armor units weight of the main layer, and with this value the remaining elements of the section, underlying layers, core and, in case, wave wall are calculated. In case of the vertical or composite breakwater, the calculation is realized applying a semi-empirical law of maximum pressures and subpressures (Goda 1985; Takahashi 1996), and the breakwater dimensions are the necessary to delimit the failure by slide and overturn- This procedure has many weak points. First of all, the formulations and laws that are applied were obtained by certain experimental set-ups. Secondly, in many occasions, these set-ups cannot be reproduced faithfully in prototype for not having the equivalent materials. Thirdly, the transposition of experimental results to prototype has scale effects (Pérez-Romero et al., 2009), modifying the partition of the incident energy, the characteristics of the flow in front of the breakwater (result of the interaction between the incident and reflected wave) and, in consequence, the actions on the breakwater and its response.

With the current knowledge there is no a methodology that allows the design and verification of other different typologies from the three traditional ones, or the quantification of the changes in the breakwater behavior by using other materials or changing the geometry of the section.

For several years the "Grupo de Dinámica de Flujos Ambientales" of the University of Granada has been working to quantify the behavior of various breakwater typologies designs as geometrical evolutions of the three traditional ones, Figure 1, and in particular, in the incident energy partition. An important conclusion has been derived from all of these studies: energy distribution is the variable which gives more information about the structural behavior of the dike, independently of the breakwater typology.

Therefore, the objective of this work is to study the variables involved in a composite breakwater behavior as a function of the dike geometry and wave characteristics for irregular waves and to obtain a methodology to calculate the loads over the structure.
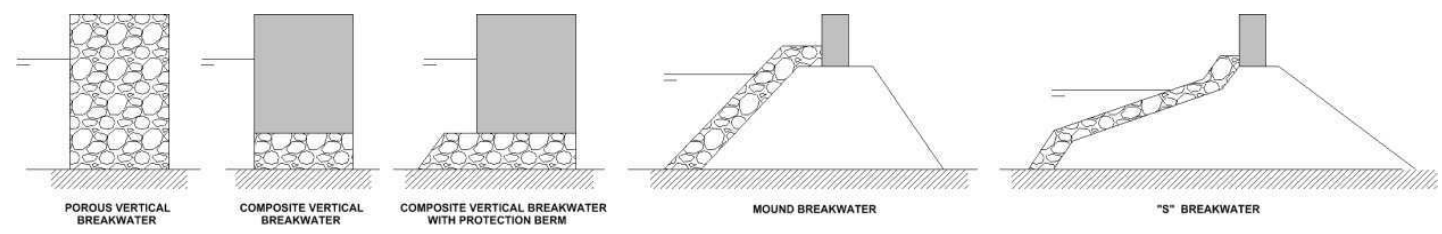

Figure 1: Breakwater typologies tested

\begin{abstract}
1 Grupo de Dinámica de Flujos Ambientales, Universidad de Granada, Centro Andaluz de Medio Ambiente. Avda. del Mediterráneo s/n. 18006 Granada, Spain Emails: mclavero@ugr.es, mvilchezsolis@ugr.es, mlosada@ugr.es.
\end{abstract}

2 PROES Consultores S.A., C/ General Yagüe, 39, 28020, Madrid, Email: dperez@proes.es, ibenedicto@proes.es 


\section{BACKGROUND}

The previous works done in the "Grupo de Dinámica de Flujos Ambientales" of the University of Granada have described the behavior of a permeable vertical breakwater (Scarcella et al. 2006; PérezRomero et al. 2009), and a composite breakwater (Pérez et al. 2010) interacting with regular waves.

\section{Permeable vertical breakwaters}

The behavior of a permeable vertical breakwater was analyzed, showing that the wave-structure interaction depends mainly on the relative width $B / L$, the relative diameter $D k$ and the ratio diameter $D / H$, where $B$ is the width of the breakwater, $L$ the wavelength, $D$ the stone diameter, $k$ the wave number and $H$ the wave height.

For the range of relative width $B / L$ analyzed, the results showed that the reflection coefficient increases to reach its maximum value; also, there is a zone of saturation adjustment and a saturation regime where the reflection coefficient can be considered constant.

A characteristic friction diagram was obtained (Pérez-Romero et al 2009, see Figure 2) considering the wave energy balance. This allows for the quantification of the resistance to flow through the porous media. Furthermore, it intends to provide an adequate selection criterion for the experimental stones diameter to minimize scale effects in the laboratory.

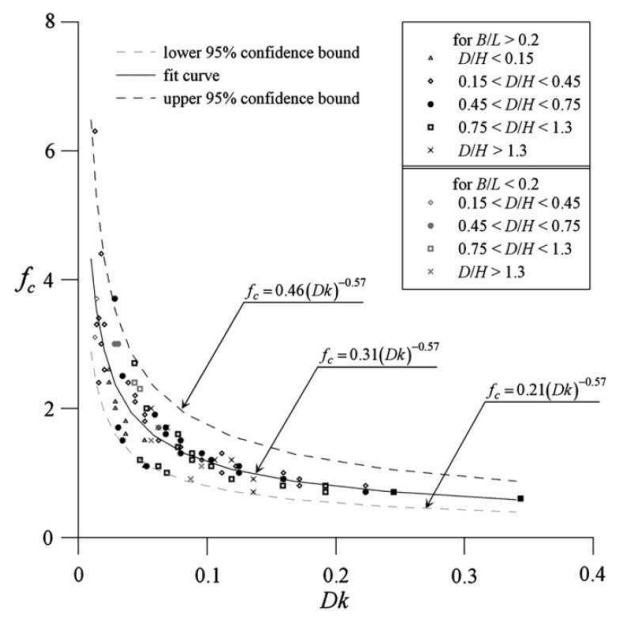

Figure 2: Characteristic friction diagram (Pérez-Romero et al 2009)

\section{Composite breakwater under regular waves action}

The analysis of the behavior of a non- overtoppable composite breakwater, with different widths $B$, foundation depths $h_{b}$ and stone diameters in the porous medium $D$, was done by Pérez et al. (2010) with regular wave trains. It was verified that the reflection coefficient increases with the breakwater relative widths $B / L$, until a maximum value from which it decreases slightly, depending mainly on the saturation regime on the relative width.

Regarding the forces, it was noted that the maximum uplift and the horizontal forces are not always in phase, and, depending on which force predominates, three zones were identified. The relation between the dimensionless forces with the total wave height at the toe of the dike depends mainly on the reflection regime and on the relative foundation depth.

\section{LABORATORY TEST}

The model consisted of a composite breakwater with rectangular cross section (Figure 3 ). Tests were done in the wave flume of CEAMA -University of Granada $(23 \times 0.65 \times 1 \mathrm{~m})$.

Three different model widths $(B=0.14,0.50$ and $1.50 \mathrm{~m})$, two different relative foundation depth $\left(h_{b} / h=0.25\right.$ and 0.50$)$ and five different granular nominal diameter $(D=12,26,40,52$ and 110) were tested.

Over 1000 tests were conducted with monochromatic waves. Wave period was varied from $T=1-3$ seconds with increment of 0.25 seconds and for each period the wave height was varied from $H_{I}=0.04$ $0.10 \mathrm{~m}$ with increments of $0.02 \mathrm{~m}$. Water depth was kept constant and equal to $0.4 \mathrm{~m}$. Test were done with non- overtopping and non-breaking conditions. 
Eight level and pressure sensors were placed to measure free surface elevations and pressures respectively.

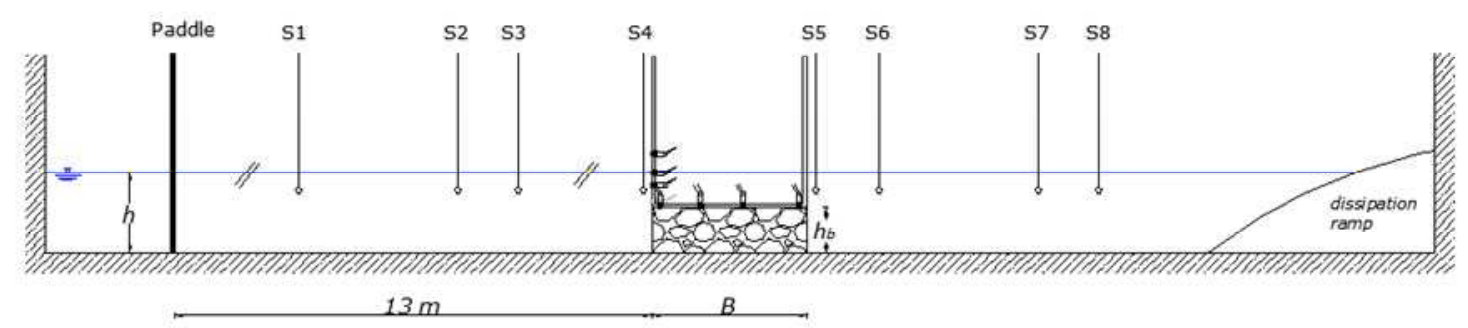

Figure 3: Description of experimental set-up

\section{ANALYSIS OF THE COMPOSITE BREAKWATER BEHAVIOR}

\section{Analysis of variables}

For each test, the following instantaneous variables were obtained:

1. Experimental reflection and transmission coefficients $\left(K_{R}\right.$ and $K_{T}$ respectively) were calculated by using two set of three sensors (S1,S2,S3 and S6,S7,S8) and applying the method proposed by Baquerizo (1995).

2. The total wave height in front of the dike $(\eta(t))$ was measured using the sensor number 4 (S4).

3. Frontal pressures $(P h(t))$ and subpressures $(P v(t))$ by using pressure sensors placed in the wall and the bottom of the caisson.

4. Horizontal and vertical forces $(F h(t)$ and $F v(t)$ respectively) were obtained integrating pressure laws.

To analyze the behavior of the irregular wave tests, the mean square value of instantaneous measured variables was obtained (Figure 4):

1. Mean square value of sea water elevation at the wall of the breakwater $\left(\eta_{r m s}\right)$.

2. Mean square value of pressure in the mean water level and pressure at the toe of the caisson ( $P_{\text {Irms }}$ and $P_{2 r m s}$ respectively).

3. Mean square value of subpressure at the entrance of the porous medium $\left(P_{\text {ulrms }}\right)$.

4. Mean square value of horizontal and vertical forces over the dike $\left(F_{\text {hrms }}\right.$ and $\left.F_{v r m s}\right)$.

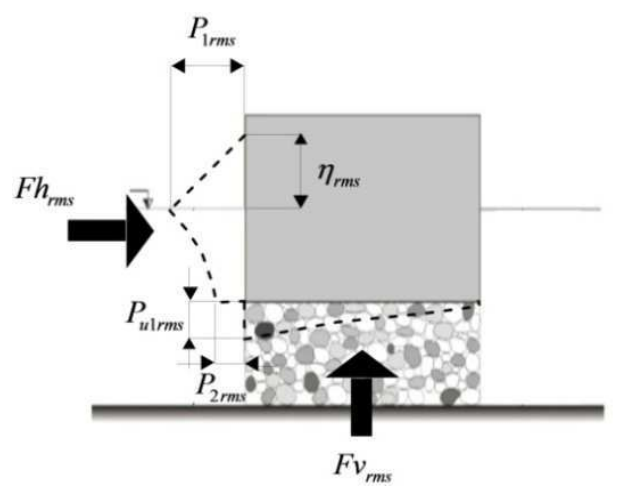

Figure 4: Analyzed variables for irregular waves

The results obtained for the analysis of these variables were:

$\underline{\text { Reflection and transmission coefficient: }}$

Figure 5 and Figure 6 shows the reflection and transmission coefficient respectively for each relative foundation depth $\left(h_{b} / h\right)$ as a function of relative width $(B / L)$. The following behavior can be observed:

1. For both foundation depth, reflection coefficient increases with $B / L$ until a maximum value (saturation point, near $B / L=0,35$ ) from which it is constant or decreases slightly. Before this point, the reflection coefficient depends on breakwater geometry and wave characteristics and when the dike reaches the saturation regime, it only depends on stone diameter or porosity. 
2. Reflection coefficient decreases when foundation depth increases because of the fact that the porous medium starts to have more importance than the impermeable wall.

3. The transmission coefficient has an opposite behavior decreasing exponentially with the increase of the relative width $B / L$.
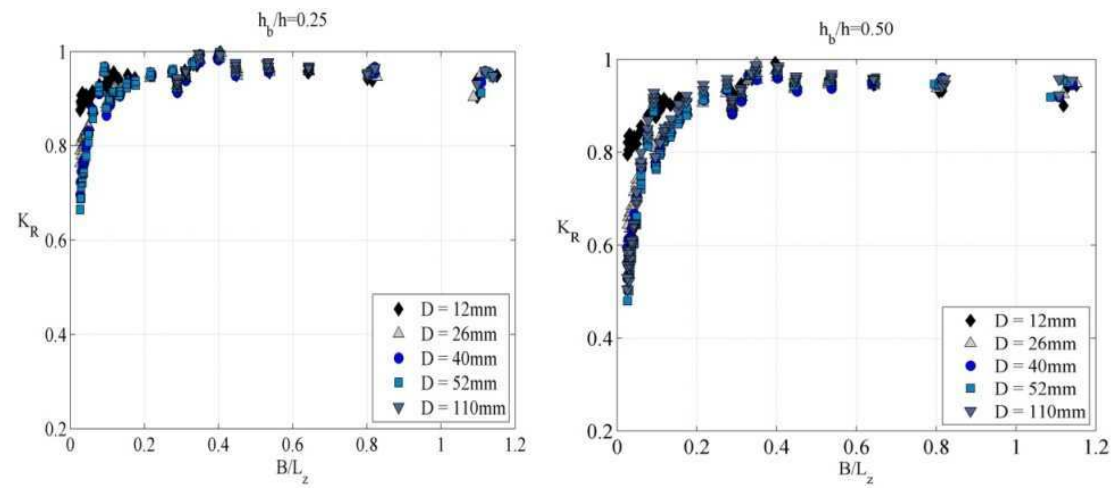

Figure 5: Behavior of the reflection coefficient
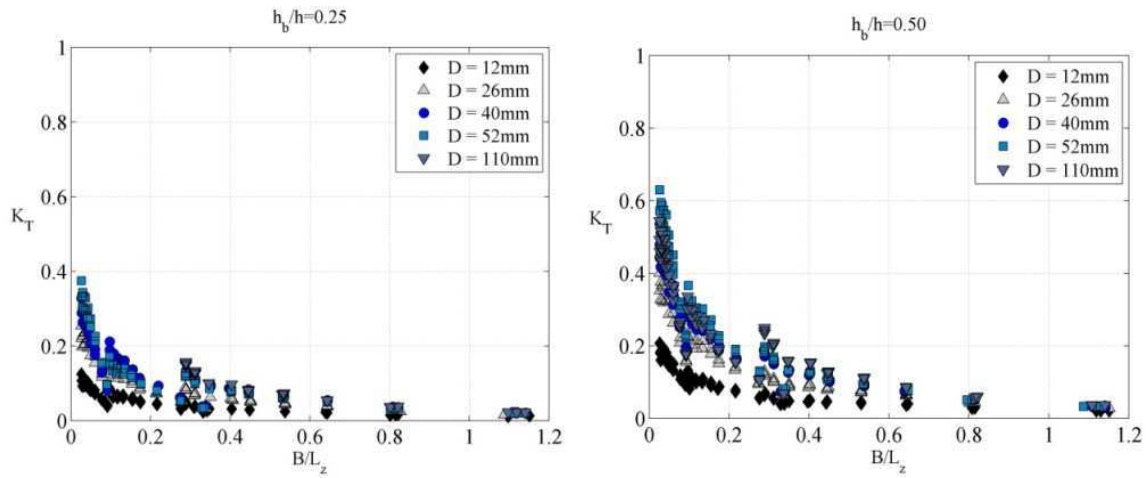

Figure 6: Behavior of the transmission coefficient

$\underline{\text { Sea water elevation at the wall of breakwater }}$

Figure 7 shows the mean square value of $\eta_{r m s}$ dimensionless with the mean square value of the total wave height at the toe of dike $\left(H_{\text {prms }}\right)$ as a function of relative depth $(h / L)$ and relative width $(B / L)$. This parameter takes a constant value around 0.6. This means that the sea water elevation at the wall of the dike depends on the total wave height at the toe of the breakwater which is due to the interaction of the incident and the reflected wave train.
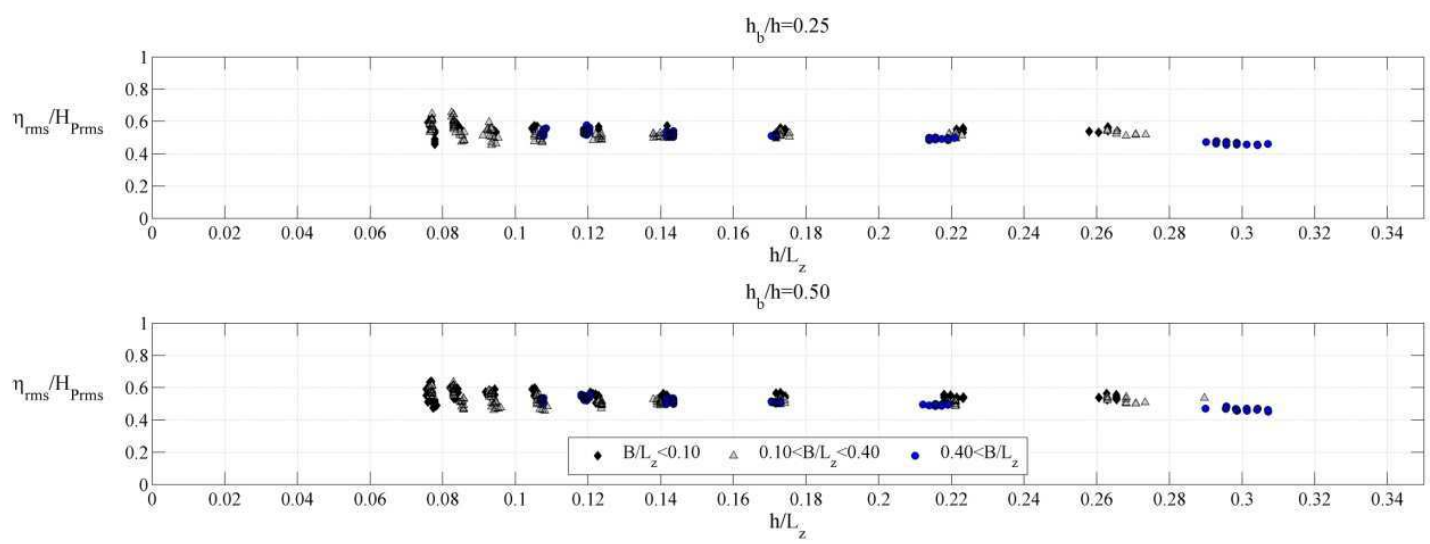

Figure 7: Behavior of the sea water elevation at the wall dimensionless with the total wave height 
Horizontal pressure

It was verified that the pressure over the mean water level is proportional to the sea water elevation at the wall as shows the following equation:

$$
P_{1 r m s}=\rho g \eta_{r m s}
$$

Where $\rho$ is the water density and $g$ is the gravity acceleration.

About pressure in the bottom of the caisson, Figure 8 shows the relationship between $P_{\text {Irms }}$ and $P_{2 r m s}$ dimensionless with wave height at the toe of the dike (which includes the reflection phenomenon). For this reason the value of $P_{\text {Irms }}$ is approximately constant. On the other hand, data is grouped in terms of $h / L$.
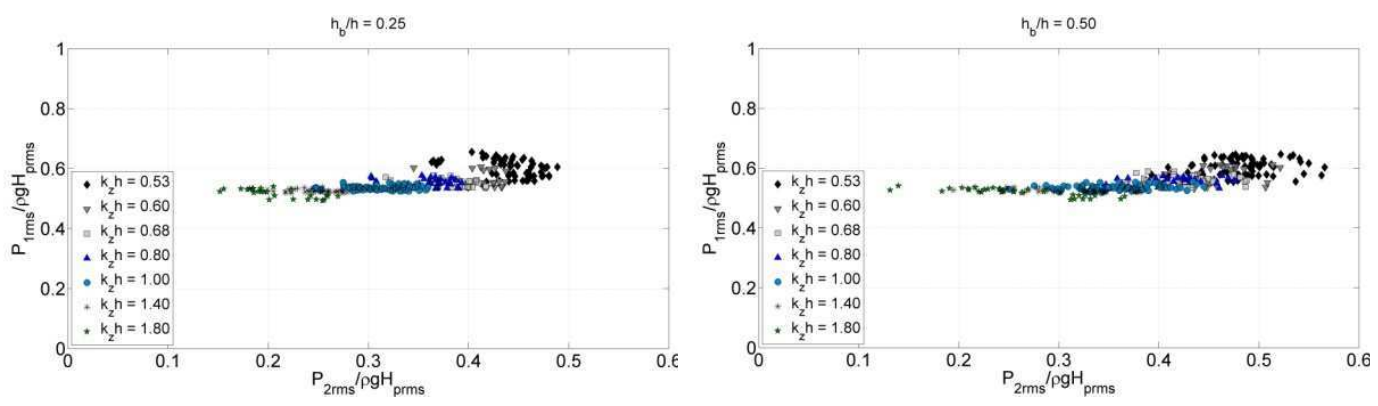

Figure 8: Relationship between pressure at the mean water level $\left(\boldsymbol{P}_{1 \mathrm{rms}}\right)$ and pressure at the bottom of the caisson $\left(P_{2 r m s}\right)$

Therefore, to obtain the pressure laws produced by the wave-breakwater interaction it is necessary to characterize the value of $\eta_{r m s}$ at the toe of the dike.

\section{Horizontal and vertical forces}

Figure 9 shows the relationship between the mean square value of horizontal and vertical forces dimensionless with total wave height at the toe of the breakwater for each relative foundation depth. These values have been plotted as a function of relative width. The main results are:

1. Data is grouped in $B / L$ intervals with linear behavior.

2. In all cases, the slope of the line is growing with $B / L$ until saturation zone and from this point, it starts to decrease.

3. Vertical forces are increasing with increment of foundation depth because there is more flow transmission through the porous medium.
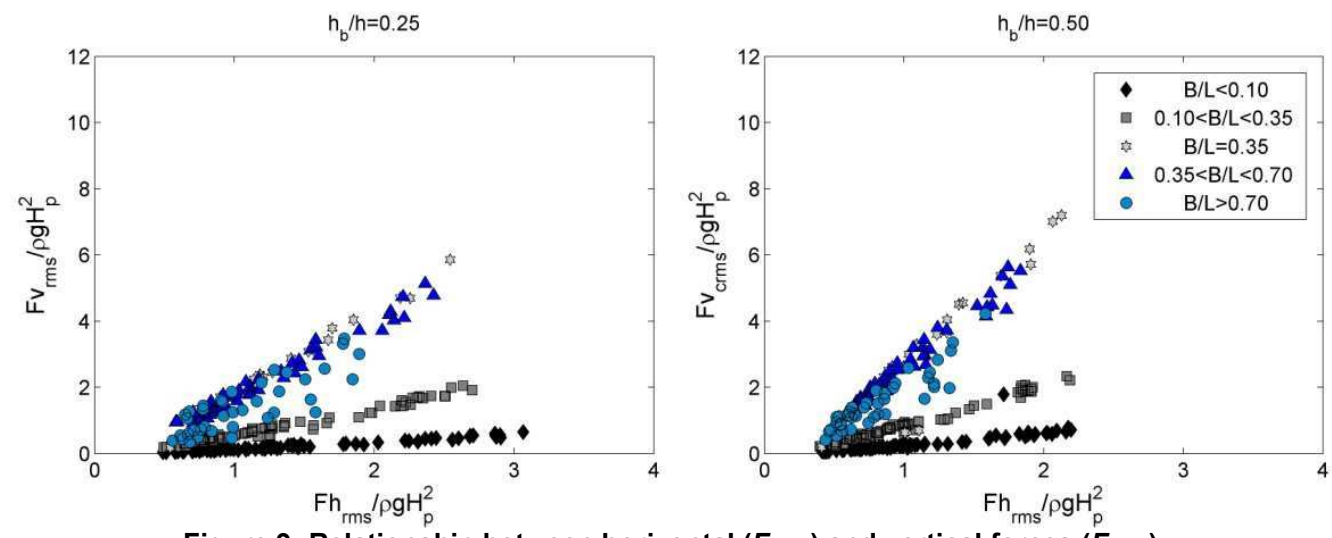

Figure 9: Relationship between horizontal $\left(F_{\text {hrms }}\right)$ and vertical forces $\left(F_{\text {vrms }}\right)$

\section{Calculation scheme}

Based on these results, it has been proved that energy distribution is the most important variable to determinate all variables involved in the breakwater stability. A simple scheme has been developed in order to calculate the loads over the structure (Figure 10). 


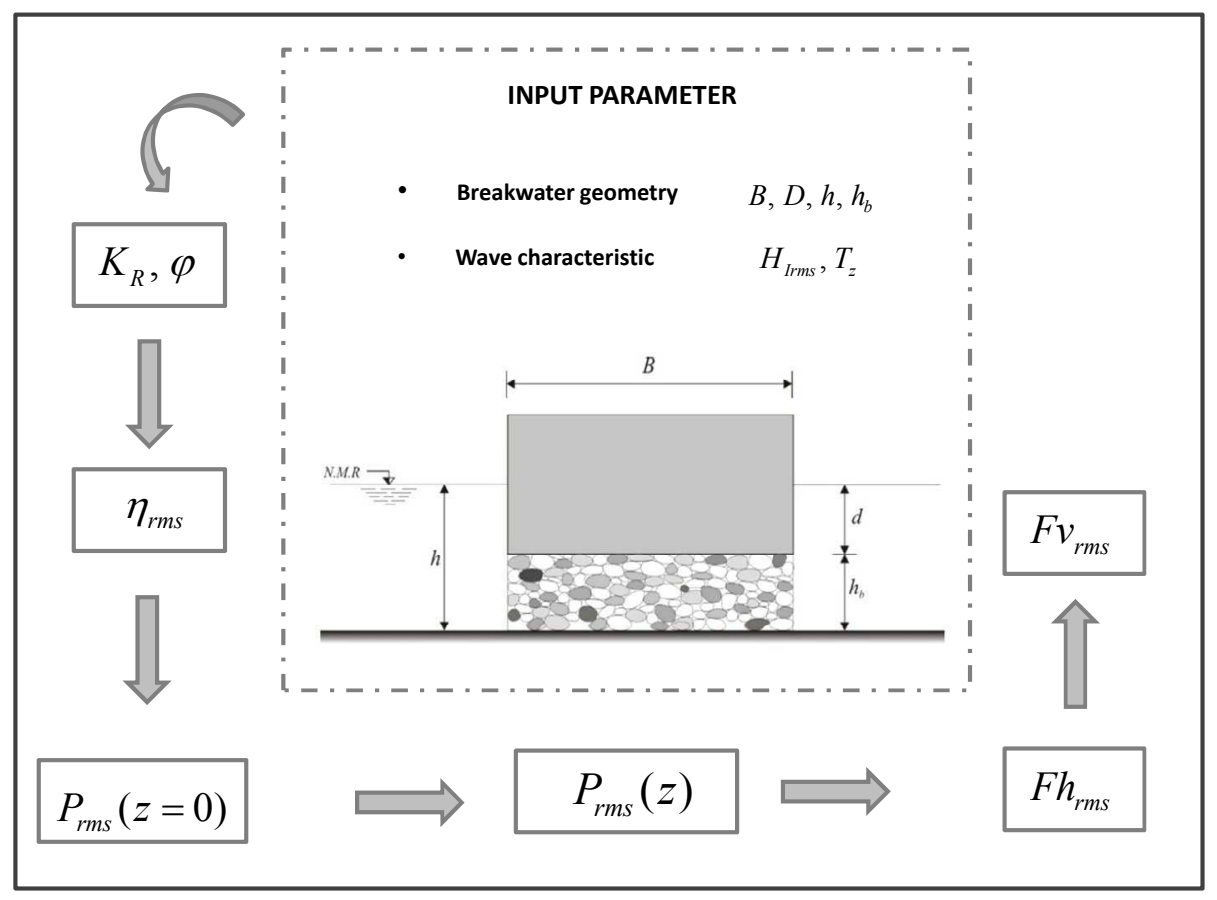

Figure 10: Scheme of calculation

In the first place, the value of the free surface at the toe of the breakwater is calculated using the next equation:

$$
\eta_{\text {prms }}=\eta_{\text {Irms }} \sqrt{1+K_{R}^{2}+2 K_{R} \cos (2 k x+\phi)}+\Delta h
$$

Where $\mathrm{k}$ is the wave number for the mean period; $\mathrm{x}$ is the distance between sensors and the toe of the dike $(\mathrm{x}=0) ; \eta_{\text {Irms }}$ is the mean square value of the incident free surface.

Reflection coefficient depends on relative width $(B / L)$, relative foundation depth $\left(h_{b} / h\right)$ and relative diameter $(D k)$. This parameter has been fitted by two curves due to the behavior observed in the Figure 5. The first curve corresponds to the range of values before saturation point $(\mathrm{B} / \mathrm{L} \leq 0.35)$, and the second curve corresponds to the range of values after saturation point $(B / L>0.35)$, (see Figure 11). The proposed fits are given in the following equation:

$$
K_{R}= \begin{cases}c_{R}\left(1+\frac{a_{R}}{c_{R}}(\tanh (B / L))^{b_{R}}\right) & \text { para } B / L \leq x_{p} \\ d_{R}(B / L)+e_{R} & \text { para } B / L>x_{p}\end{cases}
$$

where $x_{p}$ is the saturation point, and the coefficients $c_{R}, a_{R}, b_{R}, d_{R}$, and $e_{R}$ depend on relative foundation depth and relative diameter. 


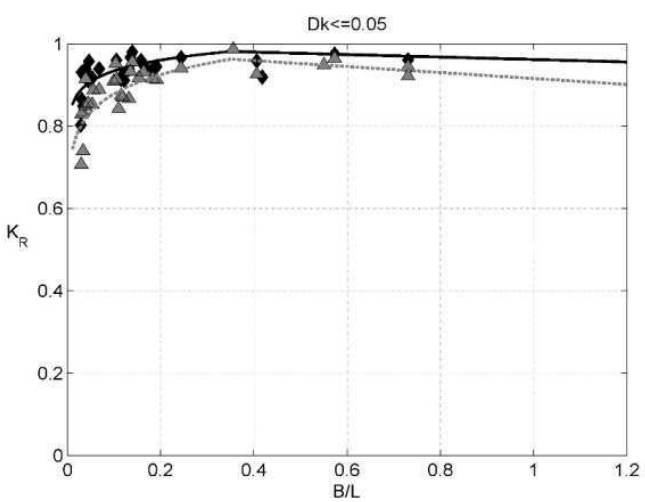

Figure 11: Reflection coefficient calculation

A non-dimensional parameter has been defined in order to obtain the value of the phase $(\phi)$ :

$$
\frac{x_{0}}{L}=\frac{\phi}{4 \pi}
$$

The phase only depends on relative depth as can be seen in Figure 12. In this figure, multiples of $2 \pi$ have been added to the measured phases to remove the confusion in presentation, due to the cyclical nature of the phase.

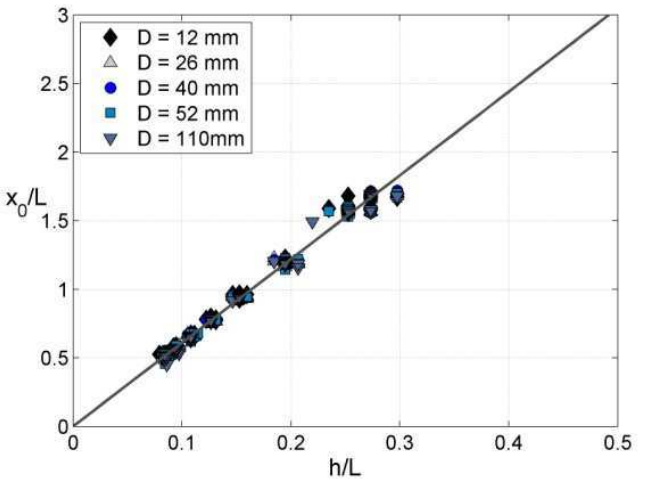

Figure 12: Phase on reflection versus relative depth

It was noted the necessity of adding a new parameter to obtain the value of $\eta_{r m s}$, the level variation $(\Delta h)$. This parameter was described by Clavero (2007). Level variation depends on wave height and relative foundation depth (see Figure 13).

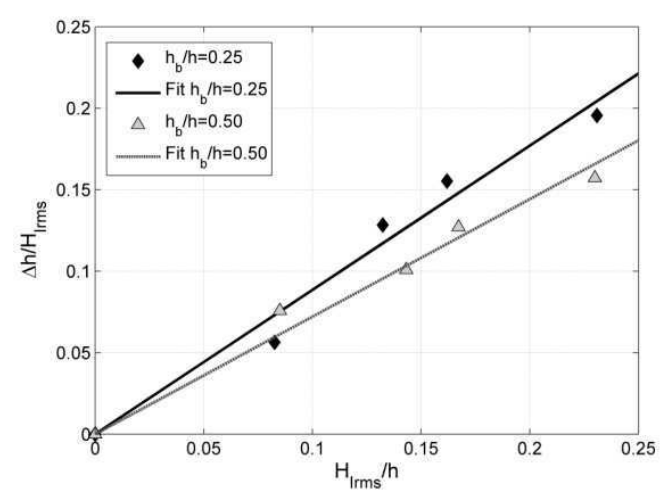

Figure 13: Level variation versus relative wave height 
When the value of $\eta_{r m s}$ has been calculated, the pressure can be obtained:

1. Pressure over mean water level is directly proportional to $\eta_{r m s}$

$$
P_{r m s}(z \geq 0)=\rho g\left(\eta_{p r m s}-z\right)
$$

2. Pressure below mean water level fits a hyperbolic cosine function for a mean period when saturation regime has been reached, and for a period around $75 \%$ of mean period when $B / L$ is minor that saturation point. Figure 14 shows an example of these results for a test before (Left figure) and other near (Right figure) of saturation zone.

$$
P_{r m s}(z \leq 0)=\rho g \eta_{p r m s} \frac{\cosh k(h+z)}{\cosh k h}
$$
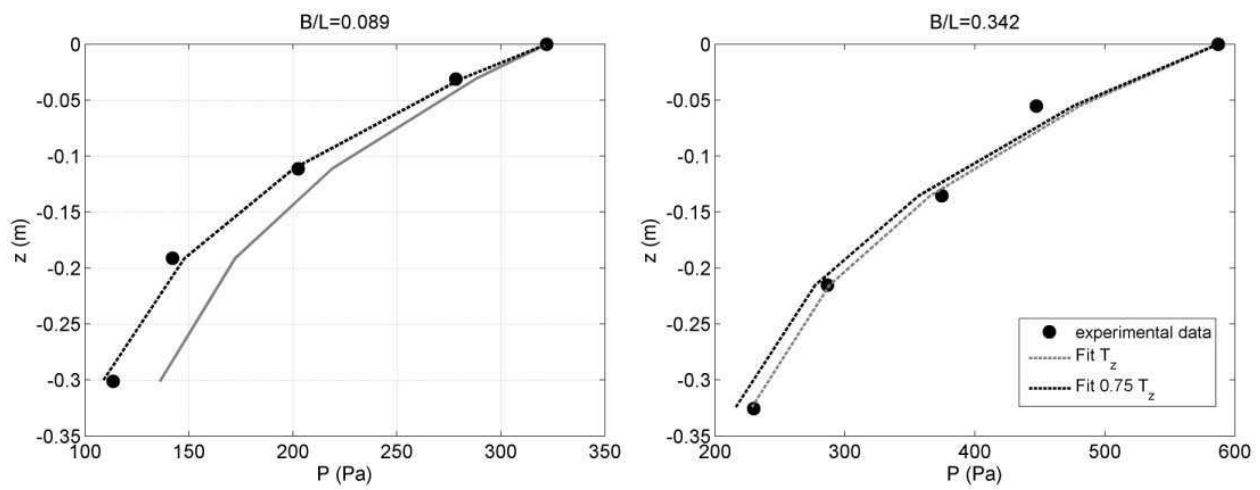

Figure 14: Pressure below reference level for a test before (left figure) and other near (right figure) saturation point $(B / L=0.35)$

The horizontal force can be obtained by integrating pressure law between the bottom of the caisson and the sea water elevation at the wall. The dimensionless vertical force can be calculated as a function of horizontal force, $B / L$ and $h_{b} / h$ (Figure 15 ) using the following expression:

$$
\frac{F v_{r m s}}{\rho g H_{p r m s}^{2}}=\beta \frac{F h_{r m s}}{\rho g H_{p r m s}^{2}}
$$
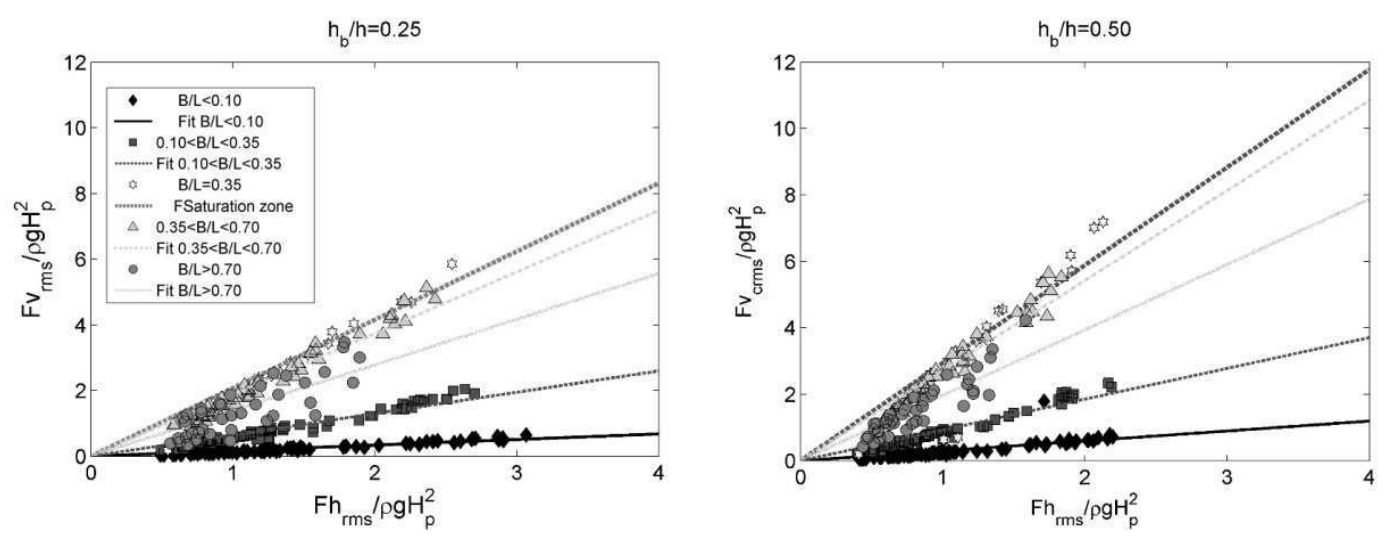

Figure 15: Vertical forces calculate 


\section{CONCLUSIONS}

Based on the results previously obtained by Pérez et al. (2010) for regular waves, the composite breakwater behavior for irregular waves has been analyzed as a function of the breakwater geometry and the incident wave characteristics. The main results show a similar behavior that for the case of regular waves:

- The reflection coefficient is defined by the saturation point, before of which it increases quickly with the relative width. The reflection coefficient takes a constant value depending only of relative foundation depth and stone diameter or porosity when saturation point is reached.

- The value of the sea water elevation at the wall and the horizontal pressure depends on the reflection coefficient.

- Horizontal pressure is directly proportional to the value of $\eta_{r m s}$.

- The relationship between horizontal and vertical forces is linear depending on the reflection regime.

The analysis of these variables shows that energy distribution is the main parameter to know the structural breakwater behavior. According with this study, a methodology to calculate the loads over the composite breakwater for regular waves has been proposed.

\section{ACKNOWLEDGMENTS}

This work was partially funded by the Spanish Ministry of Public Works (Spanish Government), project "Maritime Structures Reliability" (P50/08).

\section{REFERENCES}

Baquerizo, A., 1995. Reflexión del oleaje en playas. Métodos de evaluación y de predicción. $\mathrm{Ph}$. D. thesis, Universidad de Cantabria (Spain).

Clavero, M., 2007. Comportamiento de los diques en talud frente a la acción del oleaje y criterios para el reparto de la probabilidad conjunta de fallo en la vida útil. Phd Thesis, Universidad de Granada (Spain).

Goda Y. 1985. Random seas and design of maritime structures. University of Tokio Press

Losada, Miguel A., \& Gimenez-Curto, Luis A. 1979. The joint effect of the wave height and period on the stability of rubble mound breakwaters using Iribarren's number. Coastal Engineering, 3, 7796.

Pérez Romero, D. M., M. Ortega-Sánchez, A. Moñino and M. Losada, 2009. Characteristics friction coefficients and scale effects in oscillatory porous flow. Coastal Engineering 56, pp. 931-939.

Pérez, D., M. Correa, M. Ortega-Sánchez, M. Clavero, M. A. Losada. 2010. Pressure distributions on a vertical breakwater: experimental study and scale effects. Proceedings of 32 nd Conference on Coastal Engineering, Shanghai, China.

Scarcella, D., M. I. Benedicto, A. Moñino, M.A. Losada, 2006. Scale effects in rubble mound breakwaters considering wave energy balance. Proc. 30th Int. Conf. on Coastal Engineering. San Diego, pp. 4410-4415.

Takahashi, S., 1996. Desing of Vertical Breakwaters. P. And Harbour Research Institute. M.T., Japan

Van der Meer, Jentsje W. 1992. Stability of the seaward slope of berm breakwaters. Coastal Engineering, 16(2), 205-234. 